\title{
An evaluation of mental health clinical pharmacist independent prescribers within general practice in remote and rural Scotland.
}

BUIST, E. MCLELLAND, R., RUSHWORTH, G.F., STEWART, D., GIBSONSMITH, K., MACLURE, A., CUNNINGHAM, S., MACLURE, K. 
An evaluation of mental health clinical pharmacist independent prescribers within general practice in remote and rural Scotland

Elizabeth Buist, Rebecca McLelland, Gordon F Rushworth, Derek Stewart, Kathrine GibsonSmith, Andrew MacLure, Scott Cunningham, Katie MacLure

\section{Abstract}

\section{Background}

A 12-month pilot was implemented in two general practices in remote and rural Scotland, with patients referred by general practitioners to specialist mental health pharmacist independent prescribers.

\section{Objective}

The objective was to evaluate the pilot service from the perspectives of the patients and the care team.

\section{Methods}

The pharmacists routinely recorded patient-specific data of all clinical issues and their actions at the time of each consultation. Further datasets comprised baseline and follow-up Patient Health Questionnaire (PHQ-9) and/or Generalised Anxiety Disorder (GAD-7) rating scales, a patient survey and interviews with members of the care team.

\section{Results}

Of the 75 patients, two thirds ( $n=47,62.7 \%)$ were referred with a diagnosis of mixed depression and anxiety. There were 324 consultations (median 3, IQR 2-5, range 1-14) and 181 prescribing actions. At pilot completion, 34 patients (45.3\%) had PHQ-9 and/or GAD-7 scores reduced by $50 \%$. Patient questionnaires and staff interviews generated positive responses.

\section{Conclusion}

This pilot has provided evidence that specialist mental health pharmacist independent prescribers delivered quality care to patients with diagnoses of moderate to severe depression and/or anxiety. Whilst accepting study limitations, there is potential to translate the pilot model of care to sustained services throughout general practice. 


\section{Keywords}

Anxiety disorders, depressive disorders, pharmacist, independent prescriber, primary care, research evaluation, Scotland

Impact on Practice Statements

- Specialist mental health pharmacist independent prescribers can provide quality care for patients with anxiety and/or depression in general practice

- There is potential to translate this model of care to sustained services throughout general practice

\section{Introduction}

The role of the pharmacist within the general practice setting is developing at pace. These advances are supported by an international evidence base of systematic reviews and metaanalyses demonstrating positive effects on medication use and clinical outcomes $[1,2]$. These outcomes which are enhanced when pharmacists are fully integrated within the practice team [2]. There is also evidence of the effectiveness, safety, acceptability and positive experiences of pharmacist prescribing in a range of conditions $[3,4]$. However, the evidence for pharmacists in general practice managing patients with mental health conditions is weaker, with the only studies originating from the United States $[5,6]$.

A 12-month pilot service was implemented in two general practices in remote and rural areas of Scotland. Patients were referred by general practitioners (GPs) to specialist mental health pharmacist independent prescribers (EB, RM) working within the practices who could prescribe, within their competence, the same range of medicines as physicians. Box 1 describes the service specification. As part of the service, all patients completed Patient Health Questionnaire (PHQ-9) and/or Generalised Anxiety Disorder (GAD-7) rating scales at theira first and last appointments. 


\section{Aim of the study}

This aim was to evaluate the pilot service from the perspectives of the patients and the care team.

\section{Ethics approval}

The study was approved by the ethics review panel of the School of Pharmacy and Life Sciences at Robert Gordon University, United Kingdom. As a service evaluation, the study was exempt from National Health Service ethical and management approvals.

\section{Methods}

The evaluation was conducted by an independent research team with no involvement in service design or delivery. The pharmacists routinely recorded patient-specific data of all clinical issues and actions at the time of the patient consultations. On completion of the pilot all patients were mailed a questionnaire which had been pre-tested for face and content validity, think aloud testing and piloting. Items were derived from the CARE (Consultation And Relational Empathy) Measure [7], to obtain feedback on the consultation, items on pharmacist prescribing, and on overall experience, accessibility, setting, and demographics.

The pharmacists and members of the multidisciplinary team with whom the pharmacists had interacted were invited to participate in a semi-structured telephone interview. Written informed consent was obtained from all prior to the interviews, which were conducted by experienced qualitative researchers, audio-recorded and transcribed verbatim. The Framework Approach to data analysis was used, with codes independently collated into key themes and sub-themes by two researchers.

\section{Results}

Eighty-nine patients were referred by the GPs, 75 of whom (84.3\%) attended their initial consultation. Mean patient age was 40.1 years $(S D=13.9)$ and two thirds $(n=49,65.3 \%)$ were female. Reasons for referral were to monitor the response to treatment ( $n=25,33.3 \%$ ), review antidepressant due to lack of effectiveness ( $n=24,32.0 \%)$, discuss choice of treatment for new presentations $(n=16,21.3 \%)$, review antidepressant as unable to tolerate 
$(n=8,10.7 \%)$ and to stop antidepressant $(n=2,2.7 \%)$. Two thirds $(n=47,62.7 \%)$ were referred with a diagnosis of mixed depression and anxiety, followed by depression ( $n=22,29.3 \%$ ), anxiety $(n=3,4.0 \%)$, low mood related to bereavement $(n=1,1.3 \%)$, emotionally unstable personality disorder (borderline type) $(n=1,1.3 \%)$ and post-traumatic stress disorder $(n=1$, 1.3\%). At the first pharmacist consultation, just over one quarter $(n=20,26.7 \%)$ had PHQ-9 scores indicative of severe depression and just under half ( $n=36,48.0 \%)$ GAD-7 scores indicative of severe anxiety.

Three hundred and twenty-four consultations were held (median 3 per patient, IQR 2-5, range 1-14). Eighteen patients (24.0\%) missed one or more appointments (median 1, IQR 11 , range 1-3). There were one hundred and eighty prescribing actions, the most common being increasing the antidepressant dose $(n=77,42.5 \%)$ and starting antidepressants (or other pharmacotherapies) ( $n=59,32.6 \%)$. Medications commenced were sertraline $(n=15$, $25.4 \%)$, fluoxetine $(n=13,22.0 \%)$ mirtazapine $(n=12,20.3 \%)$, venlafaxine $(n=8,13.6 \%)$, zopiclone $(n=5,8.5 \%)$, citalopram $(n=3,5.1 \%)$, duloxetine $(n=2,3.4 \%)$ and diazepam $(n=1$, $1.7 \%)$.

Patient status on study completion is given in Table 1. Just under half of the patients $(n=34$, 45.3\%) had PHQ-9 and/or GAD-7 scores reduced by 50\% compared to their first consultation with the pharmacist. Sixteen patients $(21.3 \%)$ had not attended their appointments and were lost to follow-up.

\section{Insert Table 1 here}

Fifteen of the 70 patients ( 3 had left the practice and 2 were excluded due to terminal illness) returned the questionnaire (response rate $21.4 \%$ ). Responses to items in the CARE measure (e.g. feeling at ease, being listened to, concerns being understood etc.) were extremely positive with almost all patients giving a rating of excellent or very good across all items. Responses to items on pharmacist prescribing were also very positive in terms of pharmacists prescribing as safely as GPs (100\% strongly agree), multidisciplinary working (100\% strongly agree) and recommending consulting a pharmacist to others (93.3\% strongly agree). Textual comments on aspects of the consultation used terms such as 'felt more at ease', 'took time to get to know me', 'really listened' and 'immediately felt comfortable'. 
The three key themes which emerged from the qualitative interviews conducted with the two pharmacists, two consultant psychiatrists, three GPs and one practice manager were around integration, enablers and barriers. Emphasis was placed on the willingness of practice staff to embrace service redesign and integrate the pharmacists within the practice team. The change aligned to the new GP contact in Scotland, was accepted by patients, 'they were on board quite quickly as well...' and was perceived to not negatively impact GP workload, 'it didn't hugely change my workload...' Enablers included the service being an enhancement to standard models of care, which included prescribing by a trained and skilled professional, 'I could see it being a positive that she was able to prescribe'. Barriers appeared to be less of an issue, revolving around space constraints in the practice and initial delays in access to the electronic health record and prescriptions. There was concern that the service may not be continued on completion of the pilot, 'I have actually had consultations with a couple of patients...they were gutted that she [pharmacist] was not around anymore because they were finding it really helpful: very, very positive feedback'.

\section{Discussion}

Acknowledging study limitation, the findings of this evaluation demonstrate that the pharmacists delivered quality care to patients with diagnoses of moderate to severe depression and/or anxiety. Patients and GPs clearly trusted and valued the pharmacists' expertise and care, all of which are markers of effective implementation and service delivery [8]. The pharmacists were fully integrated within the practices i.e. full access to clinical information and referral systems, a key factor associated with improved outcomes [2]. Furthermore, as independent prescribers, the pharmacists could practise autonomously by initiating and altering medication without necessarily referring back to the GPs.

The pilot care model aligns to several key Scottish Government strategies. The Mental Health Strategy 2017-2027 has ambitions to transform services so that every general practice has multidisciplinary teams to support and treat patients with mental health issues [9]. Achieving Excellence in Pharmaceutical Care, launched in 2017 by the Scottish Government [10], describes the strategic direction for pharmacy practice in Scotland over the next decade. Emphasis is placed on developing the role of clinical pharmacist independent prescribers, with particular consideration on remote and rural settings. Pilot 
findings are therefore relevant and likely to have impact on professional practice and patient care in Scotland and beyond.

There are several limitations to this pilot hence the findings should be interpreted with caution. This is an uncontrolled study conducted in two general practices in Scotland hence the findings may not be generalisable or transferrable to other practices. The questionnaire results may be affected by response and social desirability biases. Further work to translate the pilot model of care to sustained services throughout general practice is warranted. Further to the pilot, funding has been received for a 'Teach and Treat' model whereby mental health specialist pharmacists will train non-specialist pharmacists working in practices in the effective management of moderate to severe depression and anxiety. Additionally, as a result of the pilot, an ongoing pharmacotherapy service has been funded to: support the development of pharmacy teams to be more autonomous in dealing with and managing patients with common mental health conditions; and provide a specialist mental health clinical pharmacist advisory/information service for all primary care clinicians.

\section{Conclusion}

This pilot has provided evidence that specialist mental health pharmacist independent prescribers delivered quality care to patients with diagnoses of moderate to severe depression and/or anxiety. Whilst accepting study limitations, there is potential to translate the pilot model of care to sustained services throughout general practice.

\section{Funding}

Funding for the pilot service was provided by the Scottish Government's Primary Care Transformation Fund. Funding for the evaluation was provided by the Highland Pharmacy Education \& Research Centre.

\section{Conflicts of Interest}

The authors of the evaluation have no conflicts to declare. 


\section{Acknowledgements}

The authors would like to acknowledge all participants who took part this pilot. We also thank Thom Shaw, Karen Macaskill, Michael Perera, general prcatitioners and practice staff at Glen Mor and Alness/Invergordon Medical Practices, Lochaber and Easter Ross; consultant psychiatrists and community mental health teams for their support and encouragement. 


\section{References}

1. Tan EC, Stewart K, Elliott RA, George J. Pharmacist services provided in general practice clinics: a systematic review and meta-analysis. Res Social Adm Pharm 2014;10:608-622.

2. Hazen AC, de Bont AA, Boelman L, Zwart DL, De Gier JJ, de Wit NJ, et al. The degree of integration of non-dispensing pharmacists in primary care practice and the impact on health outcomes: A systematic review. Res Social Adm Pharm 2018;14:228-240.

3. Weeks G, George J, MacLure K, Stewart D. Non-medical prescribing versus medical prescribing for acute and chronic disease management in primary and secondary care. Cochrane Database Syst Rev 2016;11:CD011227.

4. Jebara T, Cunningham S, MacLure K, Awaisu A, Pallivalapila A, Stewart D. Stakeholders' views and experiences of pharmacist prescribing: a systematic review. Br J Clin Pharmacol 2018;84:1883-1905.

5. Harms M, Haas M, Larew J, DeJongh B. Impact of a mental health clinical pharmacist on a primary care mental health integration team. Mental Health Clinician 2017;7:101-105.

6. Herbert C, Winkler $\mathrm{H}$. Impact of a clinical pharmacist-managed clinic in primary care mental health integration at a Veterans Affairs health system. Mental Health Clinician 2018;8:105-109.

7. Mercer SW, Maxwell M, Heaney D, Watt G. The consultation and relational empathy (CARE) measure: development and preliminary validation and reliability of an empathybased consultation process measure. Fam Pract 2004;21:699-705.

8. Anderson C, Zhan K, Boyd M, Mann C. The role of pharmacists in general practice: a realist review. Res Social Adm Pharm, 2019;15:338-345.

9. Scottish Government. Mental Health Strategy 2017-27 - a 10 year vision. Available from: http://www.gov.scot/Publications/2017/03/1750/0 [Accessed June 2019].

10. Scottish Government. Achieving Excellence in Pharmaceutical Care. Available from: http://www.gov.scot/publications/2017/08/4589 [Accessed June 2019]. 
Box 1. Mental health service specification

\section{Service aim}

To improve the pharmaceutical care delivered to patients with anxiety disorders and/or depression

\section{Service objectives}

1. To provide evidence based psychopharmacological interventions

2. Ensure that all prescribing is evidence based, reflecting the NHS Highland formulary

3. Ensure that the transformation of service includes multidisciplinary team working within primary care, and there is good communication with CMHTs and secondary care

4. Reduce GP workload relating to the treatment of mental health disorders

\section{Pharmacists}

The pharmacists providing the service are: registered as pharmacists and independent prescribers with the General Pharmaceutical Council; have postgraduate qualifications in Clinical Pharmacy; and more than ten years of experience working in mental health.

\section{Patient referral criteria}

Patients aged 18-65 years with a diagnosis within the last 12 months of:

- moderate to severe major depressive disorder (without psychotic symptoms)

- recurrent depressive disorder, current episode moderate to severe (without psychotic

symptoms)

- generalised anxiety disorder

- mixed anxiety and depressive disorder

- patients with the above diagnoses prescribed antidepressants long term.

- patients with any co-morbid psychiatric disorder including active substance misuse, those with immediate risk of suicide or harm to self, or to others, pregnant patients and those with post-natal depression were excluded from the service.

\section{Developmental work}

The following was carried out prior to commencing the service:

- pharmacists received training in STORM (Skills Training On Risk Management) assessment and The Decider Skills (proactive mental health cognitive behavioural therapy)

- arrangements were made for pharmacist NHS prescription pads and prescriber numbers, consulting space, computer purchase/access, appointment template

\section{Pharmacist consultations}

With advice from general practitioners and consultant psychiatrists, consultation duration was set at 30 minutes. All patients completed Patient Health Questionnaire (PHQ-9), Generalized Anxiety Disorder (GAD-7) rating scales at the first and last appointments. Prescribing was in accordance with national guidelines for the management of depression and anxiety disorders and with the NHS Highland formulary. At each consultation, the effectiveness of treatment was assessed, as were medicines related risks (e.g. adverse effects, adherence) and those relating to the clinical diagnosis (e.g. suicide risk). Oral and written information was provided in relation to use of medicines, sleep hygiene and self-help strategies. Where appropriate, specific Decider Skills were discussed with individual patients.

Table 1. Patient status on completion of the pilot study $(n=75)$

\begin{tabular}{|l|l|}
\hline Patient status & n (\%) \\
\hline PHQ-9 and/or GAD-7 scores reduced by 50\% & $34(45.3)$ \\
\hline
\end{tabular}




\begin{tabular}{|c|c|}
\hline PHQ-9 and/or GAD-7 scores not reduced by $50 \%$ & $5(6.7)$ \\
\hline Lost to follow-up & $16(21.3)$ \\
\hline Appointment times not convenient so could not attend & $3(4.0)$ \\
\hline Referred to GP for onward referral to community mental health team & $3(4.0)$ \\
\hline Referred to GP as physical health issue more dominant & $3(4.0)$ \\
\hline Psychotropic treatment not needed & $3(4.0)$ \\
\hline Appointments cancelled & $2(2.7)$ \\
\hline Psychology/ cognitive behavioural therapy to be commenced & $2(2.7)$ \\
\hline Follow-up by psychiatrist/ community psychiatric nurse & $2(2.7)$ \\
\hline Could not complete PHQ-9 and/or GAD-7 & $2(2.7)$ \\
\hline
\end{tabular}

